http://dx.doi.org/10.5902/1984686X38329

\title{
Consideraciones teóricas em torno a la educación de los sordos: especial, bilingüe, inclusiva
}

\author{
Considerações teóricas sobre a educação de surdos: especial, bilíngue, \\ inclusiva
}

Theoretical considerations about the education of the deaf: special, bilingual, inclusive

\section{Leonardo Peluso}

Profesor agregado doctor en la Universidad de la República, Montevideo, Uruguay. leonardo.peluso@gmail.com

ORCID - https://orcid.org/0000-0003-3797-6309

Recebido em 28 de maio de 2019

Aprovado em 16 de setembro de 2019

Publicado em 22 de outubro de 2019

\section{RESUMEN}

El presente trabajo pretende mostrar una perspectiva teórica e histórica sobre la educación de los sordos en Uruguay y colocar el debate que existe entre la educación especial, la propuesta bilingüe y la perspectiva de inclusión en el marco de los derechos lingüísticos de los sordos. Para hacer este análisis se parte de dos supuestos teóricos: a) las lenguas de señas son sistemas verbales y los sordos constituyen una comunidad lingüística; b) la textualidad diferida es una forma de producción textual que se puede realizar mediante dos tecnologías: la escritura y las grabaciones. El primer supuesto aleja a los sordos de la discapacidad, por lo que se opone a la idea de que las lenguas de señas sean artefactos o estrategias para compensar una falta. El segundo recoloca la relación de los sordos con las tecnologías de la lengua y habilita a considerar que éstos conforman cultura letrada mediante las visograbaciones de textos en su lengua. Los datos de los que parte este trabajo para hacer tal discusión corresponden a los resultados de investigaciones que se vienen realizando desde el año 2010 en Uruguay en la educación pública.

Palabras clave: Estudios surdos; Educación bilingüe; Textualidad diferida.

\section{RESUMO}

O presente trabalho pretende expor uma perspectiva teórica e histórica sobre a educação de surdos no Uruguai e apresentar o debate que existe entre a educação especial, a proposta bilíngue e a perspectiva da inclusão no marco dos direitos linguísticos dos surdos. Para realizar esta análise parte-se de dois pressupostos teóricos: a) as línguas de sinais são sistemas verbais e os surdos constituem uma comunidade linguística; b) textualidade diferida é uma forma de produção textual que pode ser realizada a partir de 
http://dx.doi.org/10.5902/1984686X38329

duas tecnologias: pela escrita e por gravações. O primeiro pressuposto afasta os surdos do lugar da deficiência por se opor à ideia de que as línguas de sinais são artefatos ou estratégias para se compensar uma falta. O segundo recoloca a relação dos surdos com as tecnologias da língua e permite considerar que eles estão constituindo uma cultura letrada por meio de gravações visuais de textos em sua língua. Para esta discussão, o presente trabalho teve como base resultados de pesquisas que vem sendo realizadas desde 2010 no Uruguai na educação pública.

Palavras-chave: Estudos surdos; Educação bilíngue; Textualidad diferida.

\section{ABSTRACT}

In the framework of the linguistic rights of the Deaf, the present article intends to present a theoretical and historical perspective about the education of the Deaf in Uruguay and to show the debate that exists between the special education, the bilingual proposal and the perspective of inclusion. To make this analysis, we start from two theoretical assumptions: a) sign languages are verbal systems and the Deaf constitute a linguistic community; b) deferred textuality is a type of textuality that can be made through two technologies: writing and recording. The first assumption removes the Deaf from the disability area, opposing to the idea that sign languages are artifacts or strategies to compensate a lack. The second assumption changes the relationship of the Deaf with the language technologies and allows to consider that the Deaf can generate literate culture through the visual recordings of texts in their language. The data from which this work departs to make such discussion comes from research carried out since 2010 in the Uruguayan public education system.

Keywords: Deaf studies; Bilingual education; Deferred textuality.

\section{Introducción}

El presente trabajo pretende discutir, a nivel teórico, el reconocimiento o desconocimiento de los derechos lingüísticos de los sordos en las diferentes modalidades educativas propuestas para esta población en Uruguay. Para realizar esta discusión se describirán las diferentes modalidades educativas que se han desarrollado en Uruguay y se reflexionará a partir de dos supuestos teóricos: las lenguas de señas son sistemas verbales y los sordos realizan cultura letrada en su lengua de señas a través de las visograbaciones.

Los datos utilizados para realizar tal discusión corresponden a los resultados de actividades de investigación y de extensión que se vienen realizando desde el año 2010 en el Área de Estudios Sordos, Facultad de Humanidades y Ciencias de la Educación, Universidad de la República (Uruguay), en conjunto con algunas escuelas y liceos 
http://dx.doi.org/10.5902/1984686X38329

(instituciones secundarias) que atienden a estudiantes sordos en la órbita de la educación pública uruguaya.

Estas investigaciones analizaron la legislación uruguaya y los documentos oficiales, así como también entrevistas y observación participante de quienes, por su inserción institucional, estaríamos en condiciones de realizar una historización de los procesos ocurridos en los niveles pre-universitarios de la educación pública de los sordos.

Por el tipo de temática tratada, esta discusión articula, explícitamente, aspectos teóricos, datos empíricos y una mirada política. Creemos que este es el camino que, en la actualidad, deben seguir las actividades de investigación y de extensión que pretendan ubicarse en el interdisciplinario campo de los Estudios Sordos.

\section{Las lenguas de señas son lenguas}

Desde mediados del siglo XX la lingüística de las lenguas de señas y otras disciplinas sociales y humanas sostienen que las lenguas de señas son lenguas (STOKOE, 1960/1993; BEHARES; MOTEGHIRFO; DAVIS, 1987; PARASINIS, 1999; URUGUAY, 2001, 2008/2009; PELUSO, 2010; LODI, 2014; VAL, 2016; DE LEÓN, 2019; ASUR/CINDE, 2007, solo para mencionar los más relacionados a la actividad académica uruguaya). Sin embargo, como señalaré a continuación, esta afirmación no ha sido, mucha veces, realmente comprendida.

Las lenguas de señas son sistemas verbales porque cumplen con las propiedades que históricamente la lingüística ha manejado para establecer los límites del objeto lengua. Básicamente se trata de un sistema digital de unidades; es decir, de un sistema conformado por unidades discretas que, mediante un conjunto de reglas, se pueden combinar en unidades mayores. Son sistemas productivos y creativos, al tiempo que son capaces de ponerse a sí mismos como su propio objeto (metalenguaje). La diferencia entre lenguas de señas y lenguas orales queda circunscripta a la materialidad en la que se constituyen sus significantes. Hecho que, si bien tiene claros efectos en las formas en que sus hablantes interactúan con el mundo, y en las formas en que se desarrollan las relaciones entre las lenguas y las culturas, no parece tener ninguna relevancia en el plano de los mecanismos básicos y primarios del lenguaje.

Cuando se sostiene que la lengua de señas es una lengua, esta debe quedar despojada de toda relación con la discapacidad o la falta. La lengua es una entidad de 
una naturaleza diferente a la naturaleza de los cuerpos o de las relaciones sociales, si bien, obviamente, produce efectos sobre éstos en la medida en que es constitutiva de nuestra identidad/subjetividad. Sin embargo, en la actualidad existen un sinnúmero de discursos que relacionan lengua de señas con discapacidad al referirse a los sordos. Claro ejemplo de esto es el sistema legal uruguayo, en el que en la propia ley que reconoce a la lengua de señas uruguaya como la lengua de las comunidades sordas del país, se relaciona dicha lengua con la discapacidad (URUGUAY, 2001; PELUSO, 2016; PELUSO; LARRINAGA; LODI, 2017). Esta relación de las lenguas de señas con la discapacidad desnaturaliza su carácter verbal, al tiempo que degrada dicha lengua a una ortopedia, a un artefacto necesario para compensar una falta, o para tender puentes entre discapacitados y normales. Otro ejemplo de esto es la educación. Si bien se articulan programas bilingües para los sordos, como veremos más adelante, muchas veces la lengua de señas no ingresa como una lengua sino como un recurso didáctico útil y necesario para mejor impartir los contenidos curriculares de la educación formal (PELUSO; LODI, 2015).

\section{Textualidad diferida}

Por textualidad diferida se entiende aquella textualidad que es dislocada de su momento de enunciación (PELUSO, 2015, 2018). Se trata de una textualidad que se produce para ser comprendida en un contexto diferente del cual se produjo. Para que esto pueda ocurrir, esta textualidad debe estar afectada por una tecnología de la lengua, es decir, por una tecnología que tiene por objetivo sacar al texto de su contexto de enunciación, y con ello transformarlo en un objeto físico independiente del enunciador. Esta tecnología hace que el texto diferido se diferencie de aquel que, por no ser diferido, queda inmerso en el contexto conversacional.

La característica de los textos diferidos es la de ser permanentes, objetivados (en tanto objetos físicos) e interpretables. Por permanente se entiende que, a diferencia de la oralidad que es evanescente, el texto diferido perdura más allá del momento de su enunciación (ONG, 1982; SAMPSON, 1996). Esto permite que el texto pueda ser leído en independencia de las coordenadas témporo-espaciales en las que fue enunciado. Son textos objetivados porque la tecnología de escritura o de grabación los vuelve objetos físicos, pasibles de ser manipulados, lo que no ocurre con los textos orales que, por su 
http://dx.doi.org/10.5902/1984686X38329

evanescencia, son imposibles de manipular (SAMPSON, 1996). La condición de permanencia y de manipulabilidad hacen del texto un objeto interpretable, es decir, los procesos de comprensión que se siguen al comprender un texto diferido ocurren en independencia de las negociaciones propias de los contextos dialógicos (OLSON, 1991, 1998). En el caso del diálogo yo tengo al otro con quien puedo negociar los significados, resolver mis dudas y aclarar las intenciones del decir (la fuerza ilocutiva). Los textos diferidos, en cambio, deben ser comprendidos en independencia física del enunciador, por lo que los procesos de negociación deben ser otros y el principal contexto de este tipo de texto es su propia sintaxis $u$ otros textos diferidos.

Por ser una textualidad permanente, objetivada e interpretable, ésta promueve las funciones metalingüísticas (OLSON, 1991, 1998); es decir, promueve la reflexión sobre las unidades de la lengua y sobre el propio texto; así como también instituye la posibilidad de planificación y de control sobre el decir (OCHS, 1979). Los textos diferidos pueden ser planificados y, luego de producidos, pueden ser manipulados para que adquieran la forma que su enunciador considera adecuada.

Como ya señalé, la textualidad diferida es producida por la aplicación de una tecnología sobre la lengua. Cuando esto ocurre, la lengua se modifica en sus estructuras y funciones. Por un lado las estructuras sintácticas y los campos léxicos se expanden para dar cabida a formas del decir que no estaban contempladas en la oralidad. En el marco de la textualidad diferida y de la cultura letrada se debe poder generar un texto que sea recuperado y comprendido fuera de las coordenadas témporo-espaciales de su enunciación. Por otro lado, aumenta el repertorio funcional de la legua, para dar cabida a lo que en nuestras culturas letradas se asocia a los contextos formales (gobierno, ley, educación, registro, literatura, sólo para mencionar algunos).

Desde esta perspectiva, todo texto diferido necesita de una tecnología para su realización. Las tecnologías de la lengua que habilitan este tipo de textualidad pueden ser de dos tipos: representacionales (escritura) o registrales (audio o visogabaciones). La diferencia entre ambos tipos de tecnología no es completamente superficial. Las tecnologías representacionales (los sistemas de escritura) operan brindado un código externo a la lengua que representa sus unidades. Por ejemplo, para el caso de la escritura fonográfica que se utiliza para diferir los textos en español, se utiliza un sistema compuesto por una treintena de figuras, cada uno de los cuales representa un fonema de 
http://dx.doi.org/10.5902/1984686X38329

la lengua (las correspondencias no son exactamente uno a uno, pero son sistemas casi paralelos). La particularidad de esta tecnología es que se internaliza y pasa a ocupar parte de los procesos de producción del lenguaje. Como plantea VYGOTSKI (1931/1995), la escritura como segundo sistema de señales (en tanto representa al primero) se internaliza volviéndose el primer sistema de señales. Se funde en el propio lenguaje y eso hace creer a sus usuarios que forma parte del mismo. Por su parte, las tecnologías registrales no utilizan un código representacional para las unidades de la legua, sino que operan grabando a los hablantes enunciado sus textos. Esto imposibilita una total abstracción con respecto al autor, dado que queda grabado en su propio decir. Esta tecnología, por sus características, no puede ser internalizada de la manera en que lo es la escritura, por lo que no forma parte de los procesos de producción del lenguaje de similar manera que la escritura. Asimismo, en el estado actual de desarrollo de las tecnologías de audio o visograbación, su manipulabilidad es de menor grado, así como también la promoción de la función metalingüística. Las actividades de subrayado, de tachado, de borrado, de modificación y de planificación, sencillas en la actualidad para la escritura, son complicadas para las visograbaciones. Asimismo, por ser un sistema que representa las unidades de la lengua, la escritura ya promueve en sí misma la actividad metalingüística, dado que hace evidente dichas unidades. No ocurre así con las visograbaciones, porque en éstas lo que se promueve es fundamentalmente la reflexión del nivel textual.

Debido a que la escritura era la única tecnología que, hasta el siglo pasado, permitía el desarrollo de la textualidad diferida, persiste aún hoy una tendencia a creer que escritura y textualidad diferida son lo mismo. En este trabajo sostengo lo contrario: textualidad diferida es un concepto más amplio que escritura, porque abarca otras tecnologías además de ésta.

Así, para el caso de las nuevas culturas pluritecnológicas, y fundamentalmente para el caso de los sordos cuya lengua no tiene escritura, se vuelve clave distinguir entre textualidad diferida y escritura y se torna necesario mostrar que existen otras tecnologías, de reciente invención, que también posibilitan construir textos para ser manejados fuera de su momento de enunciación.

Cualquier tipo de textualidad diferida puede habilitar la existencia de una cultura letrada si se dan dos condiciones: 
http://dx.doi.org/10.5902/1984686X38329

- La generalización de la tecnología mediante la cual se genera la textualidad diferida. Este hecho ha ocurrido en los últimos años. En la actualidad casi todos los habitantes de Uruguay cuentan con cámaras de registro auditivo y visual, en diferentes dispositivos electrónicos. Es una tecnología de acceso casi universal en la actualidad y el Estado uruguayo se ha preocupado porque los habitantes, con mayores dificultades de acceso a esta tecnología, la obtengan a través de programas gubernamentales.

- La intención de los hablantes de que los textos diferidos perduren. Esto conduce al desarrollo de modalidades de archivo. Para los textos visograbados, hoy en día la internet se ha vuelto el lugar privilegiado para realizar su acopio y archivo en diferentes sitios: Páginas Web, Blogs, Facebook, Youtube.

\section{La educación de los sordos: diversas modalidades}

Existen dos grandes modalidades en la educación de los sordos, con diversas formas al interior de las mismas: la modalidad oralista y la modalidad bilingüe. Prefiero llamarle modalidades y no momentos, porque si bien hay un derrotero histórico involucrado en el pasaje de una modalidad a otra (básicamente por el desarrollo científico y por la lucha política de los sordos), no ha ocurrido que se migre completamente de un modelo al otro. Por el contrario, tenemos un escenario educativo cada vez más complejo, muy conflictivo, en el que los diferentes modelos compiten entre sí, anudando posturas éticas, políticas y científicas que involucran a los diferentes actores (profesionales de la educación, estudiantes, comunidad sorda, familias, profesionales de la salud y científicos).

\section{Modalidad oralista}

El oralismo parte por desconocer a la lengua de señas como lengua, al negarle su carácter verbal (el primer supuesto de partida que asumo en este trabajo). Obviamente que la negación del primer supuesto de partida, conlleva necesariamente a la negación del segundo, es decir, ni se enteran de que los sordos, al tiempo que hablan una lengua de señas como primera lengua, están realizando textualidad diferida y cultura letrada en su lengua a través de las grabaciones y la internet.

La negación del carácter verbal de las lenguas de señas deja a los sordos en el marco de la falta. Se construye a los sordos como discapacitados auditivos y su identidad pasa a girar en torno al déficit. La patologización de los sordos, y la imposibilidad de 
verlos como hablantes nativos de su lengua de señas, tiene como efecto directo que los errores lingüísticos que realicen (en el sentido del apartamiento individual respecto a la norma de la lengua sonora) sean vistos desde una perspectiva clínica, no como errores de habla no nativa: se les impone, así, la patología de lenguaje.

A nivel educativo esto tiene por efecto la constitución de un modelo en el que el objetivo de enseñanza se centra en la imposición del español oral y escrito como primera y única lengua; y en la represión de todo contacto con la lengua de señas. La máxima preocupación del modelo oralista, aún más que la propia transmisión de contenidos curriculares y que la formación integral de sus estudiantes, es que los sordos se acerquen al modelo lingüístico provisto por los oyentes, a quienes llama de normooyentes. Se terapeutizan los procesos pedagógicos: los educadores se transforman en terapeutas del lenguaje y los estudiantes en enfermos a rehabilitar.

El modelo oralista tiene dos versiones: el oralismo ingenuo y aglutinante y el oralismo integrador y dispersante.

Oralismo ingenuo y aglutinante. Se trataba de una propuesta educativa en la que los estudiantes sordos eran reunidos en una escuela perteneciente a la Educación Especial (Área de la educación pública que atiende a los discapacitados) y se trabajaba con ellos el método oral (imposición de la lengua sonora como única lengua), al tiempo que se reprimía todo contacto con la lengua de señas. El único equipamiento tecnológico con el que se contaba era el audífono y como método pedagógico la clave fitzgerald, el entrenamiento auditivo para trabajar con los restos auditivos y el entrenamiento articulatorio a efectos de leer los labios y poder articular fonemas.

Lo llamo aglutinante porque ponía a todos los sordos juntos (inclusive había sistemas de internado donde los sordos se podían alojar) e ingenuo porque, al conectar a los sordos entre sí, la escuela se tornaba, paradojalmente, en un centro privilegiado de transmisión de la lengua de señas y de la cultura sorda. Al estar los estudiantes sordos todos juntos, se constituía comunidad sorda en torno a la lengua de señas, aunque en la clandestinidad.

Este oralismo desapareció en Uruguay, no sin muchas contradicciones y conflictos, entre mediados de los ochenta y mediados de los noventa del siglo XX, cuando se comenzó a migrar hacia el modelo bilingüe e intercultural que rige en la actualidad en todas las escuelas públicas para sordos y clases especializadas. 
http://dx.doi.org/10.5902/1984686X38329

Oralismo integrador y dispersante. La versión actual del modelo oralista propone que los estudiantes sordos sean equipados mediante un implante coclear, se los rehabilite lingüísticamente $\mathrm{y}$, tratados como oyentes, se los integre en escuelas en las que no comparten el salón de clases con otros sordos. Es un modelo claramente organizado por los médicos que realizan implantes cocleares y los técnicos rehabilitadores que los acompañan, que cuenta con el beneplácito de ciertos sectores educativos que se someten al discurso/poder médico y de familias que, en su ignorancia, creen que sus hijos se volvieron oyentes $y$, por lo tanto, temen el contacto con otros sordos, a quienes ven peligrosos en su poder contaminante.

Es un modelo integrador dispersante porque coloca a los estudiantes sordos aislados de otros estudiantes sordos, cada uno en clases con estudiantes oyentes. Se eliminan así las clases especiales y se profundiza la idea de que ningún estudiante sordo debería estar en contacto con otro, bajo el argumento de que tal contacto perjudicaría el desarrollo de la lengua sonora por la comodidad que ofrece la lengua de señas. Bajo la excusa de los derechos y de la igualdad, el discurso integrador dispersante, en el caso de los sordos, pisotea los derechos lingüísticos de los estudiantes al tiempo que pretende abaratar costos en la educación.

Este modelo busca que los sordos no generen comunidad sorda y que la lengua de señas no se transmita. Se destruye, sistemáticamente, toda posibilidad de que los estudiantes sordos generen identidades contra-hegemónicas que los liberen de la patologización de su experiencia de vida y del negocio de quienes se alimentan de dicha patología.

\section{Modalidad bilingüe}

El modelo bilingüe, luego de su prohibición planetaria a partir de 1880 con el Congreso de Milán, resurge a mediados de los sesenta del siglo XX como reacción frente al oralismo (GONZÁLEZ, 2014).

Con la afirmación científica de que las lenguas de señas son lenguas (STOKOE, 1960) desde el centro mismo del poder académico (la academia norteamericana), comienza un lento proceso de retorno de las lenguas de señas a las instituciones educativas a las que asistían sordos. En Uruguay este proceso comienza a fines de los 
http://dx.doi.org/10.5902/1984686X38329

ochenta del siglo XX, dos años después de la recuperación de la democracia como sistema político.

Se podría decir que la modalidad bilingüe en la educación de los sordos ha adoptado dos formas diferentes: el bilingüismo escriturocéntrico y el bilingüismo intercultural.

Bilingüismo escriturocéntrico. El modelo bilingüe se afirma en el primer supuesto de partida de este trabajo: el carácter verbal de las lenguas de señas. Sin embargo, en este período de bilingüismo escriturocéntrico se está lejos aún de incorporar el segundo supuesto: la existencia de las visograbaciones y sus formas de archivo para la consolidación de textualidad diferida y cultura letrada en lengua de señas. Se está lejos, básicamente, porque en aquel entonces las tecnologías de grabación no se habían generalizado aún en nuestras comunidades, por lo que no tenían ninguna incidencia remarcable en las prácticas textuales de los sordos.

La entrada de la lengua de señas a las escuelas fue una revolución frente al oralismo. Colocó la perspectiva de que, para los estudiantes sordos, la lengua de señas era su lengua primera, nativa, materna y que la lengua sonora funcionaba como una segunda lengua, aún cuando fuera la lengua familiar. Al tiempo que revolucionaria, esta propuesta ofrecía un sinnúmero de problemas y desafíos.

A nivel estructural la escuela tuvo que dar cuenta del ingreso de una lengua que carecía de léxicos y gramáticas descriptivos o prescriptivos, dado que no había pasado por el proceso de estandarización. No existía una variedad estándar de la lengua de señas que se pudiera transmitir desde la institución escolar. Asimismo, era una lengua que no contaba con el desarrollo léxico relacionado con los territorios científicos, necesario para impartir con comodidad una clase sobre alguna disciplina científica. Tampoco contaba con escritura y, por lo tanto, con textos escritos en dicha lengua. Elementos todos que son el insumo clave para el trabajo en nuestras modalidades de escuela.

A nivel de estatus, la lengua sonora tenía el prestigio de la institucionalidad mayoritaria, por su vinculación con la cultura letrada, con la academia y con el poder; y la lengua de señas mantenía el prestigio de ser el patrimonio de la comunidad minoritaria y minorizada (PELUSO, 2010). Frente a esto, se reconstituyó en el plano escolar la misma distribución funcional de las lenguas y sus hablantes que existía fuera: la diglosia. La lengua sonora era tomada como la variedad alta, de prestigio, tecnologizada, con léxico 
científico, en torno a la cual existía una cultura letrada y un importante acervo textual. La lengua de señas era usada para transmitir los contenidos curriculares propuestos por oyentes y para hablar de lo comunitario. En una investigación realizada en una institución de enseñanza bilingüe para sordos, algunos estudiantes señalaron que el español to asociaban a cosas importantes, básicamente relacionadas con los contenidos curriculares, y que la LSU la asociaban a pavadas, a asuntos de la comunidad (PELUSO, 2010). Asimismo los maestros, profesores y directores eran todos oyentes y a los sordos les quedaba el lugar de auxiliar de servicio (funciones de cocina, mantenimiento, portería) o de instructores, que eran sordos a los que se les daba un pequeño curso para trabajar como acompañantes de los maestros a efectos de introducir el modelo lingüístico sordo (su rol básicamente consistía en hablar en lengua de señas con los estudiantes). En un inicio no se entendía con claridad el papel de los educadores sordos, más allá de hablar en lengua de señas y de oficiar como modelos lingüísticos nativos adultos.

La propuesta educativa tuvo varios matices, pero en el fondo se podría resumir como un modelo en el que se terminó especializando a la lengua de señas para la oralidad y a la lengua sonora para la escritura. El gran desafío teórico-metodológico de la época era cómo enseñar a escribir en lengua sonora a punto de partida de la oralidad en lengua de señas.

Esto supuso que no se introducían contenidos asociados a la cultura sorda, ni a su comunidad, al menos de forma planificada y explícita. Tampoco se colocaba a la lengua de señas como objeto de reflexión, en parte porque era, hasta ese momento, una lengua que no había pasado por un proceso de gramatización (la elaboración de léxicos y gramáticas descriptivas o prescriptivas), sin el cual es muy difícil habilitar una estructura de meta-reflexión sobre la lengua, tal como ocurre hoy en día.

Al no tener a la cultura sorda y a la lengua de señas como objetivos educativos, en este primer bilingüismo se caía en el uso de la lengua de señas como recurso didáctico. Es decir, se degradaba a dicha lengua al estatus de ser un mero artefacto compensatorio, útil para tender puentes frente a las supuestas barreras comunicacionales que impondría la sordera (tal como se menciona desde el discurso de la discapacidad), y fundamentalmente, para poder llegar mejor a los estudiantes sordos con los contenidos curriculares de los diferentes programas. 
http://dx.doi.org/10.5902/1984686X38329

Esta forma de bilingüismo mantenía con fuerza el supuesto de que la única forma de textualidad diferida para los sordos era el español escrito, por lo tanto cuando se pensaba en el letramento de los sordos se pensaba en el español escrito. Así, si bien se defendía el lugar de la lengua de señas en la educación de los sordos, como primera lengua, se seguía sosteniendo que el español escrito era el centro de la educación. Esto es obvio por la propia función de la escuela. En la medida en que, en términos generales, la función de la escuela es enseñar la escritura y las prácticas discursivas concomitantes, los sordos debían pasar por un proceso de letramento, y, en aquella época, la única posibilidad de realizarlo era en torno a la escritura de la lengua sonora. Así, se medían los buenos o malos resultados en la educación de los sordos según su aprendizaje en lectura y escritura de la lengua sonora.

Algunos investigadores que abrazaban este modelo escriturocéntrico sostenían que para salir de lugar de opresión en el que se encontraban los sordos, y para que pudieran hacer oír su voz, estos debían aprender a escribir y a leer en la lengua sonora en su variedad estándar. Esta propuesta educativa mantenía, entonces, indemne, al normoyente del oralismo como modelo lingüístico, no ya en la oralidad y la escritura, sino exclusivamente en la escritura, bajo la forma, supuestamente políticamente correcta, de la necesidad de alcanzar la variedad estándar.

Estamos así frente a una perspectiva completamente escriturocéntrica que seguía siendo, en cierto sentido, oralista, dado que continuaba colocando al modelo oyente como su objetivo primario y mantenía a la lengua de señas en un lugar degradado, en tanto mero recurso didáctico. En definitiva se seguía educando para la lengua sonora y para la cultura letrada organizada en torno a esta.

Otro gran problema de los inicios de la educación bilingüe de los sordos, fue la dificultad que tuvieron, fundamentalmente los oyentes, para migrar del paradigma médico clínico al paradigma lingüístico. Si bien se sostenía que los sordos eran hablantes de la lengua de señas, como su primera lengua y natural, no se había abandonado aún la noción clínica y patologizante de que los sordos son discapacitados auditivos o comunicacionales. Persistían ambas concepciones sin conciencia del conflicto que existe entre ellas. Esto tenía efectos sobre las prácticas educativas, dado que seguir pensando en términos de discapacidad llevaba a la construcción de un sujeto de la educación sospechado. Se ponía bajo sospecha a la lengua de señas ante sus posibilidades de 
http://dx.doi.org/10.5902/1984686X38329

vehiculizar un verdadero pensamiento abstracto y por ende se ponía bajo sospecha a sus hablantes en cuanto a las capacidades de abstracción y de desarrollo de un pensamiento académico. Bajo esa sospecha se planificaban las actividades y se trabajaba en clase.

Asimismo, por este prejuicio, se pretendía enseñar a los sordos a ser visuales, sin entender el cabal significado de esto y de su relación con ser hablantes de una lengua que organiza sus significantes en una materialidad viso-espacial, por lo que el tratamiento de la visualidad se banalizaba por completo (PELUSO y LODI, 2015),

En relación a la enseñanza de la lengua sonora, propiamente dicha, como el paradigma se mantenía en el marco de la clínica y, por ende, de la visión de los sordos desde la patología del lenguaje, los errores que se producían en dicha lengua, o las dificultades que pudieren surgir en su aprendizaje, eran vistos como patología y no como el habla de aprendiz de segundas lenguas. Esto ocurría aun cuando se sostuviera que se enseñaba la escritura de la lengua sonora a punto de partida de la lengua de señas. Se mantenía así, sin cuestionamientos, el método oralista clínico para la enseñanza de la lengua sonora, aunque era la perspectiva con la que se estaba supuestamente rompiendo.

Bilingüismo intercultural. Con décadas de práctica del modelo bilingüe de educación para los sordos, se comienza a complejizar la idea de que el español escrito sea el objetivo central de la educación de los sordos y de que la metodología de enseñanza de la escritura del español debía partir de la oralidad de la LSU. Fundamentalmente se comienza cuestionar que, para que exista una verdadera educación bilingüe e intercultural, es necesario no sólo hablar en lengua de señas, sino también, sobre la lengua de señas. Asimismo, nos dimos cuenta que muchas de las respuestas de las interrogantes que estaba colocando la educación bilingüe de los sordos no estaban en la educación, sino que debían venir de otro lado: de la propia comunidad sorda.

El modelo bilingüe escriturocéntrico logra incorporar la idea de que la lengua de señas es una lengua. Si bien lo hace con ciertas dificultades, como ya señalé, esto tuvo importantes consecuencias políticas en cuanto al reconocimiento de la lengua de señas y de los derechos lingüísticos de los sordos. El modelo bilingüe intercultural hace el segundo corte en relación a la restitución de derechos lingüísticos de los sordos: se incluye a las visograbaciones como contenido y objetivo educativo, al tiempo que la lengua de señas y la cultura sorda se instalan en posición de equidad con respecto a la 
lengua sonora. Se rompe, así, con la idea de que cuando se habla de textualidad diferida de los sordos, se habla exclusivamente del español escrito. Es decir, se vira desde una posición escriturocéntrica hacia una pluritecnológica. Se coloca a la escritura en una perspectiva más amplia, como una de las tecnologías que habilitan la textualidad diferida, junto con las visograbaciones. El letramento de los sordos pasa a entenderse, también, como un proceso bilingüe. Se trabaja con los estudiantes sordos para que estos puedan ingresar a la textualidad diferida, tanto mediante las visograbaciones en su propia lengua natural (LSU), como mediante la escritura de la lengua sonora.

Se promueve la introducción de la cultura sorda y la lengua de señas en el currículum explícito. Se rompe con la idea que sólo se deben enseñar contenidos relacionados con la cultura letrada constituida en torno al español, e ingresan la cultura sorda y la propia lengua de señas como objetos de reflexión y de análisis. A punto de partida de lo señalado anteriormente, en este modelo educativo se pasa a promover prácticas letradas en torno a la textualidad diferida en lengua de señas y se comienza a utilizar los incipientes instrumentos de endogramatización de la lengua de señas que se fueron desarrollando.

Con este cambio, la lengua de señas deja de ser tratada como un recurso didáctico y pasa a ser entendida cabalmente como una lengua. $Y$ la cultura sorda deja de estar circunscripta a los intercambios sociales entre los sordos y pasa a ser un contenido curricular: se enseña historia sorda, literatura sorda, geografía sorda. Con este cambio se le asigna el lugar lógico a esta lengua y a esta cultura en el marco de una educación que pretenda ser verdaderamente bilingüe e intercultural.

En cuanto al paradigma desde donde se ancla este modelo bilingüe e intercultural, hay un distanciamiento con respecto al enfoque clínico y una inmersión en el campo de los Estudios Sordos. Esto supone que se estabiliza la concepción de los sordos en términos de hablantes de una lengua de señas y no como personas carentes o enfermas. Se rompe con la idea de que el modelo siga siendo el normooyente y se retoman los planteos que surgen del Marco Común Europeo para la enseñanza de lengua en torno al sujeto plurilingüe como sujeto de la educación lingüística (CONSEJO DE EUROPA/ DIVISIÓN DE POLÍTICAS LINGÜÍSTICAS, 2002). Se libera a aprendices y enseñantes de segundas lenguas del modelo del hablante nativo: cada hablante se colocara dónde pueda o dónde quiera en el continuo que se abre entre las dos lenguas (LARRINAGA, 
http://dx.doi.org/10.5902/1984686X38329

2014; LARRINAGA; PELUSO, 2017). Se aparta, así, a los sordos, de la opresión y estigmatización de sus modos de escribir en español. Cada estudiante sordo buscará establecer su propia relación con el español y ubicarse en el lugar del continuo interlingüístico en el que desee o pueda estar. Teniendo en cuenta esto, las producciones divergentes de los sordos en español con respecto a la norma estándar dejan de ser vistas como errores, o como síntomas de una patología del lenguaje, y pasan a ser entendidas como producciones de habla no nativa que, inclusive, pueden tener una arista política (DE LEON; et al., 2014; DE LEON, 2019).

El cambio de paradigma, que supuso la despatologización de la experiencia sorda, no sólo saca al español de la patología del lenguaje y del modelo normoyente, como señalé, sino que además libera a la lengua de señas de la sospecha en cuanto a su potencialidad como lengua. Se empieza a entender los verdaderos alcances de sostener que las lenguas de señas son lenguas. Esto hace que también se vea como absurdo enseñar la visualidad a los sordos, en la medida en que lo visual es constitutivo de su lengua (PELUSO y LODI, 2015).

Otro aspecto que se modifica en la propuesta bilingüe e intercultural es la relación entre sordos y oyentes. Los educadores sordos pasan a tener un rol clave en el trabajo con los estudiantes con la textualidad diferida en lengua de señas, con la cultura sorda letrada que se produce en torno a esta y con la lengua de señas como objeto de reflexión. También los sordos han realizado práctica como profesores de español para otros sordos (LARRINAGA, 2018). Asimismo, a nivel de las relaciones políticas, educadores oyentes y educadores sordos renegociaron sus posiciones, evitando la reconstrucción de las relaciones de opresión, que generalmente ocurren entre oyentes y sordos, al interior de la escuela. Si bien las políticas educativas siguen estando en manos de los oyentes, los sordos cada vez tienen mayor incidencia en ese nivel. De a poco, y gracias a innumerables luchas, los sordos están pasando de ser objetos a ser sujetos de su educación.

\section{Reflexiones finales}

A lo largo de este trabajo he mostrado las diferentes modalidades que ha adoptado la educación de los sordos. La vieja discusión entre modelos oralistas y bilingües, que 
http://dx.doi.org/10.5902/1984686X38329

más que científica es política e ideológica, sigue tan vigente como hace ciento cincuenta años, salvo que ahora muestra nuevas aristas.

Desde la posición adoptada en este trabajo, la modalidad bilingüe resulta la única que parece mostrarse como respetuosa de los derechos lingüísticos de los estudiantes sordos y su comunidad, en tanto hablantes de una lengua minoritaria y minorizada. Sin embargo, no toda modalidad bilingüe cumple realmente con este propósito y, en virtud de las aparentes sutilezas de los enfoques que allí convergen, a veces queda solapada la gigantesca frontera que existe entre un bilingüismo y el otro. En estas reflexiones finales pretendo explicitar aquellos aspectos en los que me parece que un modelo de educación de los sordos debe sostenerse para que realmente sea bilingüe e intercultural.

La educación bilingüe e intercultural incorpora una discusión crítica de la relación que existe entre los sordos y la discapacidad. Un modelo bilingüe e intercultural entiende a sus estudiantes no como personas carentes de un sentido, sino como hablantes de una lengua de señas, en tanto primera, natural, materna, nativa y como hablantes de una lengua sonora en tanto segunda, no natural y fundamentalmente ligada a las prácticas de escritura. En este marco, es clave, entonces, que quienes integran este modelo discutan el lugar que tiene la discapacidad en estos contextos. Un lugar que nada tiene que ver con el cuerpo, sino con la estructura de opresión y de estigmatización en la que los sordos son colocados por las características de sus cuerpos. Es claro que una educación bilingüe e intercultural no debería estar al servicio de educar discapacitados, sino que tendría por objetivo educar a hablantes nativos de una lengua de señas e integrantes de una comunidad minoritaria que se constituye en torno a esta.

La educación bilingüe e intercultural asume una postura de inclusión social en la que queda claro que no existen vectores. El gran problema que han tenido las concepciones en inclusión social y educativa hegemónicas es que conllevan la idea de que existen grupos minoritarios a los que la sociedad mayoritaria, homogeneizada, debe incluir. En general se habla de que la sociedad debe incluir, tolerar y aceptar a los diferentes. Se sostiene una lógica de incluidos y excluidos en términos de individuos marcados, pertenecientes a grupos marcados. Por lo tanto, estamos ante una lógica que perpetúa, aparentemente sin proponérselo, relaciones de dominación, opresión, complementariedad, propia de la ideología de la normalidad. 
En este sentido, una perspectiva educativa de los sordos que pretenda tener en cuenta sus derechos lingüísticos, y que se sostenga en el marco de la inclusión social y educativa, requiere perder todo vector, sin dejar de reconocer que los sordos están, por el momento, en una situación de minoirzación y estigmatización. En este marco no se explicitan sujetos de la inclusión (ni como individuos marcados, ni como grupos marcados), sino que se reconoce la disidencia de aquellos grupos que conforman identidades políticas que exigen sean respetados en su identidad. Se migra de una inclusión transitiva, aquella que cuenta con un objeto directo (incluimos a los sordos) hacia una inclusión intransitiva, aquella que carece de objeto directo. Se trata de una perspectiva que concibe a la inclusión como cohesión social, en el sentido de que todos los grupos con identidad política pueden coexistir en equidad, sin la constitución de relaciones de opresión, dominación o desigualdad y su correlato en la naturalización de actitudes coloniales de tolerancia y aceptación. La inclusión social y educativa intransitiva busca que las personas se reconozcan y se respeten en equidad.

Para el caso de los sordos, la inclusión intransitiva exige que se deseche todo intento de integrar/incluir a los estudiantes sordos de forma solitaria en escuelas con hablantes de lenguas sonoras, anulando todo contacto con sus pares. Por el contrario, es un modelo que incluye centralmente, en el currículum escolar, el campo de los estudios sordos, la reflexión ideológica acerca de la comunidad y la promoción, en los estudiantes, de formas de reconocimiento de su identidad sorda como identidad política.

En la educación bilingüe e intercultural se entiende que la lengua de señas no es un recurso didáctico, sino el objetivo central de la misma. Esta modalidad de educación rompe con la tendencia, que se observa en muchas formas de propuesta bilingüe para los sordos, a degradar a la lengua de señas al lugar de recurso didáctico, sólo utilizada para transmitir información. Por el contrario, una educación bilingüe e intercultural asume la perspectiva de que la lengua de señas, en el caso de los sordos, es un objetivo formativo en sí mismo. Es un lugar para ser, para pensar y para pensarse. Es el espacio de confort personal y de los intercambios sociales más significativos. Es la lengua que sostiene, además, el patrimonio cultural del grupo.

La educación bilingüe e intercultural incorpora la educación lingüística en lengua de señas en contextos interculturales. La educación bilingüe e intercultural de los sordos parte de la base de que ambas lenguas y ambas culturas tienen sus propios instrumentos 
http://dx.doi.org/10.5902/1984686X38329

de análisis y de reflexión; y de que ambas lenguas y ambas culturas cuentan con textos diferidos estabilizados y archivados. Es decir que asume que en ambas lenguas se desarrolla cultura letrada, conformada por textos a los que sus hablantes pueden recurrir a efectos de interpretarlos y de reflexionar sobre sus marcos históricos, éticos y políticos.

Esto ocurre porque se rompe con la vieja dependencia del sistema de escritura para pensar en la textualidad diferida y la cultura letrada, que dejaba a la lengua de señas y a la cultura sorda en la descripción de orales, ágrafas o no-letradas. Se pasa a entender que la textualidad diferida y la cultura letrada, en el caso de las lenguas de señas y de la cultura sorda, dependen de otras tecnologías, como las visograbaciones, que también permiten la generación de textualidad diferida y cultura letrada en torno a la lengua de señas. Esto supone, además, que se elimina al oyente como modelo lingüístico de la lengua sonora y que se empieza a considerar que el español escrito es siempre una segunda lengua para los sordos, por lo que su manejo debe estar condicionado a sus particularidades. Se plantea, en términos políticos, la existencia del estilo sordo del español, que visibiliza la apropiación que hacen los sordos del español y su rechazo al modelo estándar, en tanto modelo opresor y colonial.

Teniendo en cuenta estos aspectos, la educación bilingüe e intercultural de los sordos rompe con el centralismo que históricamente ocupó el letramento en lengua sonora, donde inclusive hasta hoy se miden los éxitos o fracasos en la educación de los sordos en términos de cuánto aprendieron a leer y escribir. Dicho centralismo escriturocéntrico usa a la lengua de señas para cumplir con este propósito, olvidándose de ésta y de la comunidad y cultura sorda que se instituyen en torno a sus prácticas comunicativas. En cambio, la educación bilingüe e intercultural de los sordos, entiende que sus estudiantes y docentes son pluritecnológicos, plurilingües y multiculturales, y establece relaciones de equidad y reconocimiento entre ellos.

El objetivo central de este modelo educativo es formar ciudadanos críticos que puedan entender su historia, interpretar su cultura y tener un proyecto de vida acorde a sus propias expectativas e ideología. En ese sentido, la modalidad bilingüe e intercultural es un modelo educativo que reconoce y promueve los derechos lingüísticos y educativos de los estudiantes sordos.

En la actualidad en Uruguay ya abandonamos el modelo bilingüe hacia el plurilingüe, en el sentido que le otorga el Marco Común Europeo de Referencia para las Lenguas 
http://dx.doi.org/10.5902/1984686X38329

(CONSEJO DE EUROPA / DIVISIÓN DE POLÍTICAS LINGÜÍSTICAS, 2002). No solo se descarta la idea de hablante nativo y de lenguas instituidas en espacios separados, sino que además se entiende que los estudiantes sordos deben formarse teniendo en cuenta las características plurilingües de su comunidad: la lengua de señas uruguaya como primera lengua y natural; el español como segunda lengua y la lengua de la mayoría del país en el que viven; el inglés como lengua que lidera los procesos de globalización; y una lengua de señas internacional, en la medida en que los sordos participan de la comunidad sorda global.

Para finalizar, a lo largo de este trabajo he presentado varias modalidades de educación de los sordos y he mostrado las razones por las que la educación bilingüe (actualmente plurilingüe) e intercultural de los sordos es la única logra reconocer y promover los derechos lingüísticos y educativos de los sordos; recoger las plataformas políticas actuales de las comunidades sordas; y atender al propio desarrollo de la cultura sorda con los nuevos procesos de tecnologización de sus lenguas y de sus medios de comunicación. Esto ocurre porque es una modalidad educativa que tiene por objetivo formar sujetos críticos, plurilingües, pluritecnológicos, conocedores y transformadores de sus lenguas, conocedores de su historia, protagonistas de las decisiones de sus comunidades, orgullosos de pertenecer a la comunidad sorda, autónomos, libres, satisfechos de sí mismos y sólidamente instrumentados para pasar por todas los desafíos que ofrece la vida.

\section{Referências}

ASUR/CINDE. Diccionario Bilingüe de Lengua de Señas Uruguaya/Español. 1 ed. Montevideo: CINDE, 2007.

BEHARES, Luis Ernesto; MONTEGHIRFO, Nellys; DAVIS, Dee. Lengua de Señas Uruguaya. Su Componente Léxico Básico. 1 ed. Montevideo: Instituto Interamericano del Niño, 1987.

CONSEJO DE EUROPA / DIVISIÓN DE POLÍTICAS LINGÜÍSTICAS. Marco Común Europeo de Referencia para las Lenguas. Aprendizaje, enseñanza, evaluación. 1 ed. Madrid: Instituto Cervantes, 2002.

DE LEÓN, Adriana. Distribución funcional de las lenguas en la comunidad sorda. Consideraciones lingüísticas, tecnológicas, históricas y políticas. Montevideo: inédito, 2019. 
http://dx.doi.org/10.5902/1984686X38329

DE LEÓN, Adriana et al. Los sordos, el español escrito y la comunicación. In: TUILSUimagen Producciones 2014. Consideraciones teóricas, metodológicas y políticas. Montevideo: Ediciones TUILSU-imagen, 2014. p. 31-35.

GONZÁLEZ, Rodrigo. Historia Ilustrada de la Comunidad Sorda. De la antigüedad a nuestros días. 1 ed. Montevideo: Ediciones TUILSU, 2014.

LARRINAGA, Juan Andrés. Linguistic, educational and political aspects regarding two groups in Uruguay: Deaf students and University students. Psicología, Conocimiento y Sociedad, Montevideo, n. 4, v. 2, noviembre, 2014. Disponível em:

file:///C:/Users/hp/Downloads/231-1360-1-PB.pdf. Acesso em: 27 maio 2019.

LARRINAGA, Juan Andrés. Enseñanza de español para sordos. Consideraciones desde una propuesta plurilingüe. Borradores, Montevideo, n.3, v.4, p. 56-65, julio 2018.

LARRINAGA, Juan Andrés; PELUSO, Leonardo. Bilingual Education or Multiple Linguistic and Technological Channels Shaping a Plurilingual Education: The Uruguayan Experience. In: GERNER, Bárbara de Garcia; KARNOPP, Lodenir. (Ed.). Change and Promise. Bilingual Deaf Education, Sign Language and Deaf Culture in Latin America. Washington: Gallaudet University Press, 2017. p.171-192.

LODI, Ana Claudia Balieiro. Texto e Discurso em Libras: possibilidades de apropriação de práticas de leitura e produção de textos/discursos por alunos surdos. In: ERNST, Aracy; LEFFA, Vilson; SOBRAL, Adail. (Comp.). Ensino e linguagem. Novos desafios Pelotas: Educat, 2014. p. 97-126.

OCHS, Elinor. Planned and unplanned discourse. In: GIVON, Talamy. (Ed.) Discourse and Syntax. New York: Academic Press, 1979. p. 51-80.

OLSON, David. El mundo sobre papel. 1 ed. Barcelona: Gedisa, 1998.

OLSON, David. La cultura escrita como actividad metalingüística. In: OLSON, David y TORRANCE, Nancy. (Ed.) Cultura escrita y oralidad. Barcelona: Gedisa, 1991. p. 333358.

ONG, Walter. Oralidad y escritura. Tecnologías de la palabra. 1 ed. Buenos Aires: Fondo de cultura económica, 1982.

PARASNIS, Ilia. Cultural and Language Diversity and the Deaf Experience. 1 ed. Cambridge: Cambridge University Press, 1999.

PELUSO, Leonardo. Sordos y Oyentes en un liceo común. Investigación e intervención en un contexto intercultural. 1 ed. Montevideo: Psicolibros/UdelaR, 2010. 
http://dx.doi.org/10.5902/1984686X38329

PELUSO, Leonardo. Traducción entre español escrito y Lengua de Señas Uruguaya videograbada: un nuevo desafío. Cadernos de Tradução. Florianópolis, n. 35, vol. 2, juldez 2015. Disponível em: https://periodicos.ufsc.br/index.php/traducao/article/view/21757968.2015v35nesp2p479. Acesso em: 27 maio 2019.

PELUSO, Leonardo. La lengua de señas uruguaya y las políticas lingüísticas. Revista Virtual de Estudos da Linguagem - ReVEL -, vol.14, n.26, marzo 2016. Disponível em: http://www.revel.inf.br/files/b8ab9d9f392bbe6d21545e30d2521438.pdf. Acesso em: 27 maio 2019.

PELUSO, Leonardo. (2018). Los sordos, sus lenguas y su textualidad diferida. Traslaciones, Mendoza, vol. 5, n. 9, noviembre 2018. Disponível em: http://revistas.uncu.edu.ar/ojs/index.php/traslaciones/article/view/1311. Acesso em: 27 maio 2019.

PELUSO, Leonardo; LARRINAGA, Juan Andrés; LODI, Ana Claudia Balieiro. Public Policies on Deaf Education. Comparative Analysis Between Uruguay and Brazil. In: PINK, William; NOBLIT, George. (Org) Second International Handbook of Urban Education. Volume I. Chan: Springer, 2017. p. 613-626

PELUSO, Leonardo; LODI, Ana Claudia Balieiro. La experiencia visual de los sordos. Consideraciones políticas, lingüísticas y epistemológicas. Pro-posições, Campinas, vol. 26, n.3, setiembre/diciembre 2015. Disponível em http://www.scielo.br/scielo.php?script=sci_arttext\&pid=S0103-

$73072015000300059 \&$ Ing=en\&nrm=iso\&ting=es. Acesso em: 27 maio 2019.

SAMPSON, Geoffrey. Sistemas de escritura. 1 ed. Barcelona: Gedisa, 1996.

STOKOE, William. Sign Language Structure. An Outline of the Visual Communication Systems of the American Deaf. Silver Spring: Linstok Press,1960/1993.

URUGUAY. Ley No. 17.378. Reconócese a todos los efectos a la Lengua de Señas Uruguaya como la lengua natural de las personas sordas y de sus comunidades en todo el territorio de la República. Montevideo: IMPO, 2001. Disponível em:

https://www.impo.com.uy/bases/leyes/17378-2001. Acesso em: 27 maio 2019.

URUGUAY. Ley No. 18.437. Ley General de Educación. Montevideo: IMPO, 2008/2009. Disponível em: http://www.impo.com.uy/bases/leyes/18437-2008. Acesso em: 27 maio 2019.

VAL, Santiago Sánchez. La lingüística de las lenguas de señas como herramienta de reivindicación política: problemas ideológicos y epistemólogicos. Revista Virtual de Estudos da Linguagem - ReVEL -, vol.14, n.26, marzo 2016. Disponível em: http://www.revel.inf.br/files/0013c9544c89fb90cae89f6bf1dedcb1.pdf. Acesso em: 27 maio 2019.

VYGOTSKI, Lev Semyonovich. (1931/1995). Historia del desarrollo de las funciones psíquicas superiores. In: Obras escogidas. Tomo III. 1 ed. Madrid: Visor. p. 183-206. 
http://dx.doi.org/10.5902/1984686X38329

\section{Correspondência}

Leonardo Peluso - Universidad de la República, Calle Rivera 1350, 50000 Salto, Departamento de Salto - Uruguay.

\section{(c) (i) (8)}

This work is licensed under a Creative Commons Attribution-NonCommercial 4.0 International (CC BY-NC 4.0) 